\title{
Data Quality Challenges in Distributed Live-Virtual-Constructive Test Environments
}

JEREMY R. MILLAR, DOUGLAS D. HODSON, GILBERT L. PETERSON, and DARRYL K. AHNER, Air Force Institute of Technology

CCS Concepts: • Computer systems organization $\rightarrow$ Architectures; Distributed architectures; Real-time systems; $\bullet$ Software and its engineering $\rightarrow$ Software organization and properties; Virtual worlds software; Software system structures; Real-time systems software; Software functional properties; Correctness

Additional Key Words and Phrases: Distributed simulation, performance estimation

ACM Reference Format:

Jeremy R. Millar, Douglas D. Hodson, Gilbert L. Peterson, and Darryl K. Ahner. 2016. Data quality challenges in distributed live-virtual-constructive test environments. J. Data and Information Quality 7, 1-2, Article 2 (April 2016), 3 pages.

DOI: http://dx.doi.org/10.1145/2850420

\section{INTRODUCTION}

Live-Virtual-Constructive (LVC) simulations are complex systems comprising a combination of live (real people operating real equipment), virtual (real people operating simulated equipment or vice versa), and constructive (wholly simulated) entities. Nodes in the system support the simulation of one or more entities and are often geographically distributed to leverage unique assets (e.g., physical test range space or high-fidelity full motion simulators). Nodes are connected in a peer-to-peer fashion and communicate using protocols such as Distributed Interactive Simulation (DIS) [DIS Steering Committee 1998], the High Level Architecture (HLA) [Dahmann et al. 1997], or the Test and Training Enabling Network Architecture (TENA) [Powell and Noseworthy 2012].

Distributed LVC simulation promises a number of benefits for the test and evaluation (T\&E) community, including reduced costs, access to simulations of limited availability assets, the ability to conduct large-scale multiservice test events, and recapitalization of existing simulation investments. Consequently, the Department of Defense (DoD) is increasingly turning to LVC simulation and virtual environments to support T\&E events. LVC simulations have been used to test communications for unmanned aircraft systems [Parker et al. 2009], conduct cyber-security analysis [Van Leeuwen et al. 2010], and quantify radar measurement errors [Hodson et al. 2013].

Ensuring rigorous results for T\&E events supported by LVC simulation requires addressing three fundamental data quality challenges: quantifying numerical errors due to weakly consistent nodes, assessing measurement accuracy with respect to tolerance requirements, and assessing measurement quality in the absence of absolute truth values.

Authors' addresses: J. R. Millar, Communications and Information Directorate, Air Force Institute of Technology, 2950 Hobson Way, Wright Patterson, AFB 45433; D. D. Hodson and G. L. Peterson, Department of Electrical and Computer Engineering, Air Force Institute of Technology, 2950 Hobson Way, Wright Patterson, AFB 45433; D. K. Ahner, Department of Operational Sciences, Air Force Institute of Technology, 2950 Hobson Way, Wright Patterson, AFB 45433.

Permission to make digital or hard copies of part or all of this work for personal or classroom use is granted without fee provided that copies are not made or distributed for profit or commercial advantage and that copies bear this notice and the full citation on the first page. Copyrights for third-party components of this work must be honored. For all other uses, contact the owner/authors(s).

(c) 2016 Copyright held by the owner/author(s).

1936-1955/2016/04-ART2 $\$ 15.00$

DOI: http://dx.doi.org/10.1145/2850420 


\section{DATA QUALITY CHALLENGES}

LVC simulations are typically designed as fully replicated, geographically distributed database applications with real-time constraints. The data of interest are composed of entity and world state information and derived quantities such as collisions or weapons effectiveness. These data must be replicated at each node to meet availability and responsiveness requirements. The inclusion of live or virtual entities imposes realtime constraints on database responsiveness since long read/write latencies cannot be tolerated. Consequently, entity state updates (i.e., writes to a database record) are propagated to other system nodes after taking effect locally and are delayed due to network latency. Thus, not all nodes see the same simulation state at the same time. If updates cease, the system will eventually become consistent [Terry et al. 1994]. As such, LVC simulations can be viewed in the same context as eventually consistent distributed datastores such as Amazon's Dynamo [DeCandia et al. 2007], Cassandra [Lakshman and Malik 2009], or Megastore [Baker et al. 2011].

For any eventually consistent distributed database, a fundamental question is "How eventual is eventual?" Common measures of eventual consistency are time (how long it takes for readers to see the result of a write) and versions (how many versions old is a given read result) [Bailis and Ghodsi 2013]. For LVC simulations and other distributed virtual environments, deviation (e.g., Euclidean distance) from a "true" value is a common measure of consistency [Yu and Vahdat 2002; Aggarwal et al. 2004; Zhou et al. 2004].

Quantifying the numerical error associated with eventual consistency is a key challenge for LVC simulations. Although there is a growing body of literature characterizing the consistency of distributed databases such as Dynamo and Cassandra [Wada et al. 2011; Rahman et al. 2012; Bailis et al. 2012], these works focus on time or version staleness as the measure of consistency, feature read-heavy workloads, and are not necessarily geographically distributed. In contrast, LVC simulations are more concerned with numerical error, have a balanced read/write workload, and are distributed geographically.

During a test event, measurements may be taken at any simulation node. Inconsistencies in the replicated state are reflected as measurement errors. A second challenge lies in assessing whether each measurement error lies within a precision tolerance. This assessment must be conducted during system design to ensure the simulation is capable of meeting test requirements. Additionally, it must occur during the test execution to provide a quantification of the uncertainty associated with each measurement.

A third challenge for LVC simulations is assessing the quality of measurements without a known truth value, particularly in the discrete case [Mauve 2000]. This is especially true for derived quantities that depend on inconsistent state data such as collisions and weapons effects. In this case, each interacting node may compute a result that is correct according to its state replica and different from other interacting nodes. Furthermore, the uncertainty can vary based on the node taking the measurement.

\section{CONCLUSION}

LVC simulations enable large-scale operationally relevant T\&E events at reduced cost, provide access to limited availability assets, and recapitalize existing simulations. System architectures based on weakly consistent replicated databases and unreliable update protocols yield three fundamental challenges for data quality: quantifying error due to eventual consistency, assessing measurement accuracy with respect to desired tolerances, and assessing measurement quality in the absence of a truth value. Addressing these challenges is fundamental to ensuring the veracity and rigor of $\mathrm{T} \& \mathrm{E}$ events supported by LVC simulation. 


\section{REFERENCES}

Sudhir Aggarwal, Hemant Banavar, Amit Khandelwal, Sarit Mukherjee, and Sampath Rangarajan. 2004. Accuracy in dead-reckoning based distributed multi-player games. In Proceedings of 3rd ACM SIGCOMM Workshop on Network and System Support For Games. ACM, 161-165.

Peter Bailis and Ali Ghodsi. 2013. Eventual consistency today: Limitations, extensions, and beyond. Communications of the ACM 56, 5 (May 2013), 55-63. DOI : http://dx.doi.org/10.1145/2447976.2447992

Peter Bailis, Shivaram Venkataraman, Michael J. Franklin, Joseph M. Hellerstein, and Ion Stoica. 2012. Probabilistically bounded staleness for practical partial quorums. Proceedings of the VLDB Endowment 5, 8 (April 2012), 776-787. http://dl.acm.org/citation.cfm?id=2212351.2212359.

Jason Baker, Chris Bond, James C. Corbett, J. J. Furman, Andrey Khorlin, James Larson, Jean-Michel Leon, Yawei Li, Alexander Lloyd, and Vadim Yushprakh. 2011. Megastore: Providing scalable, highly available storage for interactive services. In Conference on Innovative Data Systems Research (CIDR), Vol. 11. 223-234.

Judith S. Dahmann, Richard M. Fujimoto, and Richard M. Weatherly. 1997. The department of defense high level architecture. In Proceedings of the 29th Conference on Winter Simulation. IEEE Computer Society, 142-149.

Giuseppe DeCandia, Deniz Hastorun, Madan Jampani, Gunavardhan Kakulapati, Avinash Lakshman, Alex Pilchin, Swaminathan Sivasubramanian, Peter Vosshall, and Werner Vogels. 2007. Dynamo: Amazon's highly available key-value store. In ACM SIGOPS Operating Systems Review, Vol. 41. ACM, 205-220.

DIS Steering Committee. 1998. IEEE Standard for distributed interactive simulation-application protocols. IEEE Standard 1278 (1998).

Douglas D. Hodson, Alex J. Gutman, Bruce Esken, and Raymond R. Hill. 2013. Quantifying radar measurement errors in a live-virtual-constructive environment to determine system viability: A case study. Journal of Defense Modeling and Simulation: Applications, Methodology, Technology (2013), 1548512913503740.

Avinash Lakshman and Prashant Malik. 2009. Cassandra: Structured storage system on a p2p network. In Proceedings of the 28th ACM Symposium on Principles of Distributed Computing. ACM, 5-5.

Martin Mauve. 2000. How to keep a dead man from shooting. In Interactive Distributed Multimedia Systems and Telecommunication Services, Hans Scholten and Marten van Sinderen (Eds.). Springer, 199-204.

Eric Paul Parker, Nadine Elizabeth Miner, Brian Peter Van Leeuwen, and James Brian Rigdon. 2009. Testing unmanned autonomous system communications in a live/virtual/constructive environment. International Test and Evaluation Association Journal (ITEA) 30 (2009), 513-522.

Edward T. Powell and J. Russell Noseworthy. 2012. The test and training enabling architecture (TENA). Engineering Principles of Combat Modeling and Distributed Simulation (2012), 449.

Muntasir Raihan Rahman, Wojciech Golab, Alvin AuYoung, Kimberly Keeton, and Jay J. Wylie. 2012. Toward a principled framework for benchmarking consistency. In Proceedings of the 8th USENIX Conference on Hot Topics in System Dependability. USENIX Association, 8-8.

Douglas B. Terry, Alan J. Demers, Karin Petersen, Mike J. Spreitzer, Marvin M. Theimer, and Brent B. Welch. 1994. Session guarantees for weakly consistent replicated data. In Proceedings of the 3rd Internatinoal Conference on Parallel and Distributed Information Systems, 1994. IEEE, 140-149.

Brian Van Leeuwen, Vincent Urias, John Eldridge, Charles Villamarin, and Ron Olsberg. 2010. Performing cyber security analysis using a live, virtual, and constructive (LVC) testbed. In Proceedings of the Military Communications Conference (MILCOM 2010). IEEE, 1806-1811.

Hiroshi Wada, Alan Fekete, Liang Zhao, Kevin Lee, and Anna Liu. 2011. Data consistency properties and the trade-offs in commercial cloud storage: The consumers' perspective. In Conference on Innovative Data Systems Research (CIDR), Vol. 11. 134-143.

Haifeng Yu and Amin Vahdat. 2002. Design and evaluation of a conit-based continuous consistency model for replicated services. ACM Transactions on Computer Systems (TOCS) 20, 3 (2002), 239-282.

Suiping Zhou, Wentong Cai, Bu-Sung Lee, and Stephen J. Turner. 2004. Time-space consistency in large-scale distributed virtual environments. ACM Transactions on Modeling and Computer Simulation (TOMACS) 14,1 (2004), 31-47.

Received August 2015; revised October 2015; accepted November 2015 